\title{
Rare case of metachronous tumor: Nasopharyngeal and colorectal carcinoma
}

\author{
DANIELA JICMAN (STAN) ${ }^{1,2^{*}}$, ELENA NICULET ${ }^{3,4}$, MIHAELA LUNGU $^{5,6^{*}}$, \\ CRISTIAN ONISOR $^{3}$, LAURA REBEGEA $^{6}$, CARMEN BOBEICA $^{3 *}$, ALINA MIHAELA ELISEI $^{7,8}$, \\ LUCRETIA ANGHEL $^{6}$ and ALIN LAURENTIU TATU ${ }^{6,7,9}$
}

${ }^{1}$ Department of Otorhinolaringology, 'Sfantul Apostol Andrei’ Emergency Clinical Hospital, 800578 Galati; ${ }^{2}$ Biomedical Doctoral School, and ${ }^{3}$ Department of Morphological and Functional Sciences, Faculty of Medicine and Pharmacy, 'Dunarea de Jos' University of Galati, 800010 Galati; Departments of ${ }^{4}$ Pathology and ${ }^{5}$ Neurology, 'Sfantul Apostol Andrei' Emergency Clinical Hospital, 800578 Galati; ${ }^{6}$ Clinical Medical Department, Faculty of Medicine and Pharmacy,

'Dunarea de Jos' University; ${ }^{7}$ Medical and Pharmaceutical Research Unit/Competitive, Interdisciplinary Research Integrated Platform 'Dunărea de Jos', ReForm-UDJG; ${ }^{8}$ Research Centre in The Field of Medical and Pharmaceutical Sciences, Faculty of Medicine and Pharmacy, Department of Pharmaceutical Sciences,

'Dunărea de Jos' University of Galați, 800010 Galati, ${ }^{9}$ Department of Dermatology,

'Sfanta Cuvioasa Parascheva' Clinical Hospital of Infectious Diseases, 800179 Galati, Romania

Received August 6, 2021; Accepted September 6, 2021

DOI: $10.3892 /$ etm.2021.10852

\begin{abstract}
The study presents an unusual case of a patient with a personal history of a rectal malignant tumor in 2013, who after a period of 6 years, was diagnosed with an advanced nasopharyngeal carcinoma, locally and regionally invasive. It is possible that the colorectal malignant tumor affected the development of the nasopharyngeal carcinoma, or the other way around, depending on the presence of genetic instabilities. These two types of malignant tumors share a series of genes
\end{abstract}

Correspondence to: Dr Elena Niculet or Dr Cristian Onisor, Department of Morphological and Functional Sciences, Faculty of Medicine and Pharmacy, 'Dunarea de Jos' University of Galati, 35 Alexandru Ioan Cuza Street, 800010 Galati, Romania

E-mail: helena_badiu@yahoo.com

E-mail: cristi.onisor@gmail.com

*Contributed equally

Abbreviations: NPC, nasopharyngeal carcinoma; EBV, Epstein-Barr virus; HLA-I, human leukocyte class I; HIV, human immunodeficiency virus; CT, computer tomography; MRI, magnetic resonance imaging; ORL, otorhinolaryngology; RNA, ribonucleic acid; SPINK-6, serine protease inhibitor Kazal-type 6; KLK, kallikrein; SIADH, inappropriate antidiuretic hormone secretion; HHM, humoral hypercalcemia of malignancy; ACTH, adrenocorticotropic hormone; DIC, disseminated intravascular coagulation

Key words: nasopharyngeal carcinoma, Epstein Barr virus, histopathology, radiotherapy, chemotherapy, surgery, genetic modification that can influence their progression, i.e., SPINK-6 and Bcl-2. The particularity of this case stems from the development of a metachronous tumor, a rectal adenocarcinoma and nasopharyngeal carcinoma, two malignant tumors with different patient prognosis and disease progression. Research needs to be continued on the multidisciplinary therapeutic management of nasopharyngeal cancer and the ways of identifying this cancer type in its early stages, considering that most patients come from a rural environment, have poor medical education, a number of comorbidities, and who frequently ignore the signs, symptoms and sometimes the treatment offered.

\section{Introduction}

Nasopharyngeal carcinoma (NPC) is a rare Epstein-Barr virus (EBV)-associated malignant tumor with a specific geographical distribution within endemic areas (Southern China, Taiwan, the Philippines, Vietnam and Northern Africa), several predisposing etiologic factors (such as smoking, salt-preserved fish and other foods, and a low diet in fresh fruits and vegetables) and a strong genetic susceptibility revealed by the high frequency of NPC among Chinese migrant patients (10- to 30 -fold higher than other populations) and among Chinese individuals born in other countries $(1,2)$.

Other risk factors that are already established for type III NPC include the Cantonese ethnicity, male sex, EBV infection, the presence of human leukocyte class I alleles (HLA-I, more specifically HLA-A2-B46 and -B17, both present in the Chinese and other Asian populations who are at high risk; HLA-B5, in Caucasians: An ethnic background that suggests the risk of a possible morphea development; HLA-A11, present in all ethnicities; B13, present in the Chinese and Tunisian populations, and $\mathrm{A} 2$, present in the non-Chinese populations) and a family history (familial clustering) of NPC (1-5). An 
important risk factor is currently being taken into consideration, i.e., poor oral hygiene; patients with infrequent/non-daily teeth brushing, multiple dental cavities and possibly individuals living with the human immunodeficiency virus (HIV) can have an increased risk of developing NPC (1,3-5).

A patient suffering from NPC can present with several signs and symptoms, including palpable neck masses, nasal obstruction with some discharge, epistaxis, headaches and various non-specific findings. This malignancy has an unfortunate prognosis, being identified late in its development, frequently when it has already metastasized, in an advanced clinical stage (over $70 \%$ of cases). Such rare malignant tumors (NPC, spiradenocarcinoma, pulmonary neuroendocrine tumors) or even ones that have high incidence rates (hepatocellular carcinoma) have aggressive behavior that translates as rapid local invasion, high rate of metastasis and recurrence and frequent recurrence. As in many cases of aggressive malignant tumors, multimodal treatment involving a multidisciplinary team seems to be an optimal approach, involving a tandem of surgery, chemotherapy, radiotherapy, or immunotherapy.

Nasopharyngeal examination consists initially of an indirect nasopharyngoscopy procedure, with subsequent direct nasopharyngoscopy with the help of a fiberoptic endoscope, and, if a tumor mass is found, it is also biopsied. If a tumor mass is suspected and a direct examination is not diagnostic, further imaging studies are needed such as computer tomography (CT) scan and/or magnetic resonance imaging (MRI) studies, a technique that offers better contrast among structures and an improved multiplanar capability, offering an improved view of the disease extension and that of the tumor margins $(1,3,6-8)$.

As far as treatment goes, NPC is a malignant tumor that is highly sensitive to radiation therapy, even though it registers high local recurrence and metastasis rates. Tumors that are locally infiltrative (advanced) benefit from a combined therapy of chemotherapy and radiotherapy, with improved survival rates and disease-free intervals $(1,2)$.

\section{Case report}

An 80-year-old hypertensive patient with right auricular prosthesis and left ear cophosis, was admitted to the otorhinolaryngology (ORL) clinic at the 'Sfantul Apostol Andrei' Emergency Clinical Hospital in Galati, in September 2019 suffering from bloody otorrhea, deteriorating hypoacusis on the right side, right hemicranias and also vertigo. The patient revealed a personal history with a surgically treated gastric ulcer and a moderately differentiated rectal adenocarcinoma diagnosed in 2013, which was surgically removed, revealing invasion in the tunica muscularis propria. Ethics approval was granted by the 'Dunarea de Jos' University's Ethics Committee with the decision number 331 from 15.04.2021. Written informed consent was provided by the patient.

In September 2019, the initial clinical diagnosis was haemorrhagic otitis media, for which the patient received a treatment course with antibiotics, antialgics, sedatives, anti-hypertensives (including $\beta$-blockers, which can have various immunologic or non-immunologic side effects) (9) and specific local treatment with aspiration, instillations and local hygienic measures. The patient was advised to avoid aggressive endauricular maneuvers and to avoid wearing the hearing prosthesis. The medical

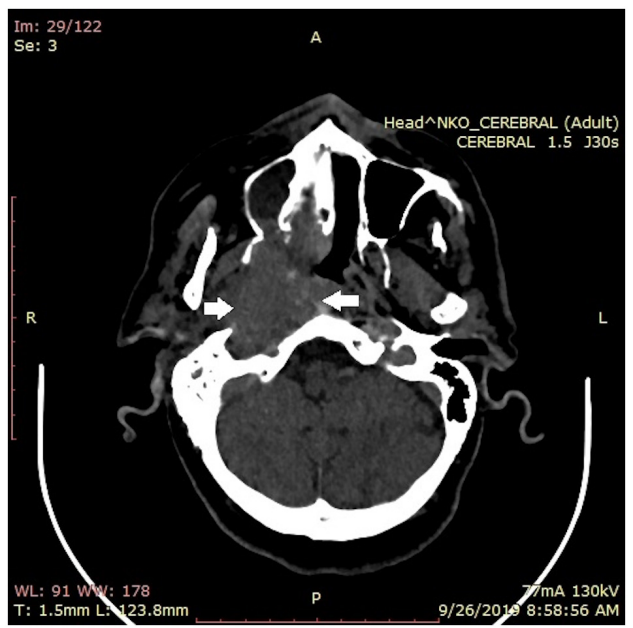

Figure 1. Computer tomography (CT) scan showing right nasopharyngeal solid tumor mass with local invasion.

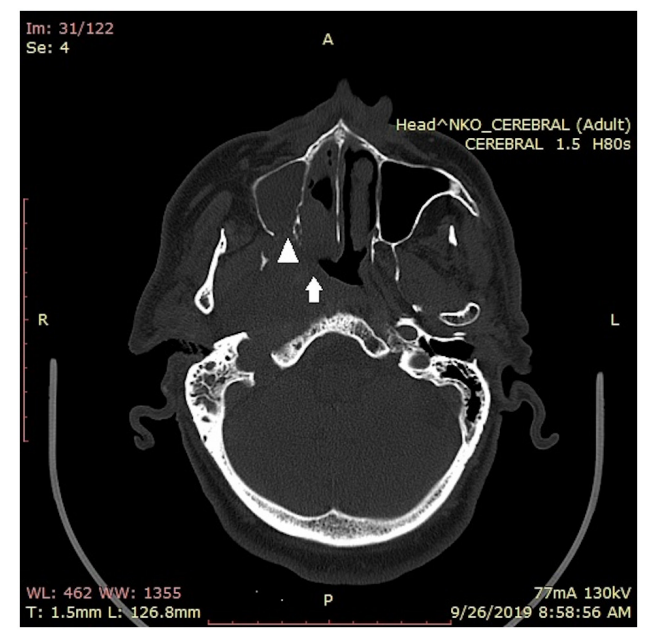

Figure 2. Computer tomography (CT) scan showing nasopharyngeal tumor mass infiltrating the maxillary sinus, right nasal fossa and the right pterygoidian fossa.

measures improved the patient's overall state by stopping the hemorrhage, but they did not relieve the hemicrania. The otomicroscopic examination revealed a perforated tympanic membrane with granulations which were further excised. The previous treatment was continued.

In September 2019, a CT scan was carried out involving the middle ear, temporal bone and brain; it revealed the presence of a 54/45 mm tumor mass located in the right infratemporal fossa with extension in the rhinopharynx, the right nasal fossa and maxillary sinus, and adjacent bone destruction and inflammation, as shown in Figs. 1-3.

The vegetant, cauliflower-like tumor mass was biopsied and the pathology report revealed it to be a poorly differentiated squamous cell carcinoma (G3). As surgical treatment was not an option, radiotherapy examination was carried out for palliative care. The pathology report, associated with the clinical and radiotherapy examination confirmed the diagnosis of a locally invasive tumor mass, with distant metastases, and a poorly differentiated squamous cell carcinoma with left cervical lymph node metastasis, a clinical stage of IVB. 


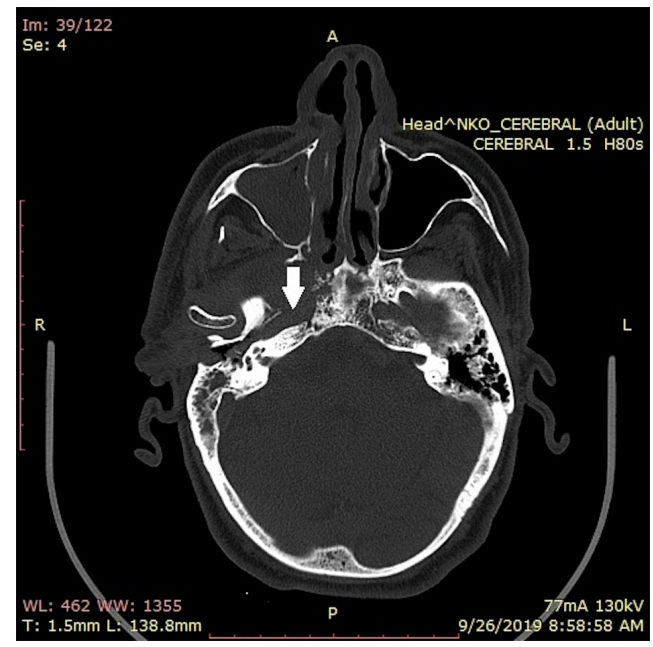

Figure 3. Computer tomography (CT) scan showing nasopharyngeal tumor mass infiltrating the anterior region of the right carotid osseous channel.

At the radiotherapist's request, an MRI examination was performed, revealing a right cavum expansive tumor process with skull base, maxilla-sphenoidal sinus, perineural, right carotid wall and ipsilateral jugular vein wall involvement, and with a right superior jugular lymph node metastasis. This led to a thoracic CT examination which found no evidence of metastatic involvement.

By taking into consideration the local and regional extent of the tumor without proof of any distant metastatic involvement, neoadjuvant chemotherapy treatment was recommended with paclitaxel-carboplatin. In March 2020, after the last evaluation due to a persistent thrombocytopenia, the patient could not undergo radiotherapeutic treatment, and in February 2021 the patient's overall state was stable.

\section{Discussion}

NPC is a distinct neoplasm among other types of head and neck cancers. By gross examination this tumor can reveal many different aspects: It can appear as a flat; smooth lesion of the mucosa; as a small, elevated nodule with or without surface ulceration; or even as a vegetant, cauliflower-like, frankly infiltrative mass, or it can also not be visible at all (10).

Most NPC histopathology studies are based on the primary tumor biopsy sample (although primary full-tumor excision would be more beneficial in patient survival, as Koebner phenomena manifested as in situ recurrence/field cancerization is frequent in oral cancers) (11-13). In many cases it is followed by cervical lymph node excision, which is frequently positive for metastases, the tumor's aggressive behavior being explained by apoptosis, proliferation and neovascularization (three processes on which the tumor's growth and metastatic capacity reside and which are promoted by interleukin-6 activity on the tumor and the immune system) $(11,14,15)$. By analyzing non-keratinizing carcinoma types, the more frequent undifferentiated subtype has a tumor growth that is characterized morphologically by a syncytial pattern of large malignant tumor cells with inconspicuous margins and vesicular oval nuclei with central, large nucleoli. These malignant cells are stacked together and sometimes the nuclei seem to have larger quantities of chromatin, with hyperchromasia, with less of a vesicular chromatin pattern (10). Low power non-keratinizing NPC biopsy examination reveals a proliferation of large epithelioid cells that are separated by layers of small lymphocytes and plasma cells, while a higher power view reveals the epithelioid component's pleomorphic nuclei with open chromatin pattern and conspicuous nucleoli. At this magnification the lymphocytic and plasma cell component is also clearly visible, and the malignant cells are positive for EBV ribonucleic acid (RNA) and following immunohistochemistry examination they are positive for CK5/6, with membrane staining having a brown pattern (16).

The keratinizing type of NPC has an obvious microscopic squamous differentiation, with intercellular bridges and keratin pearls (sometimes present as an extensive tumor component). Frequently, an in situ component is also found. This carcinoma has a development pattern characterized by tumor islands within an abundant desmoplastic stroma with a variable number of lymphocytes, plasma cells, neutrophils and eosinophils (10). Eosinophils are mainly found in the stroma in between the malignant islands, but can also be seen among the tumor cells as part of the aforementioned islands (17). This moderate inflammatory reaction to the malignant component is mostly composed of lymphocytes and polymorphonuclear leucocytes, with a reduced number of plasma cells $(11,17)$.

The epithelial component is comprised of multi-layered polygonal malignant cells having distinct cell borders and evident intercellular bridges. The cells that are found in the center of the islands and those that are located more superficially frequently have a larger quantity of glassy, eosinophilic cytoplasm, sometimes with readily identifiable cytoplasmic tonofibrils (a marker of single-cell keratinization) (11).

Although it is considered a rare type of malignancy, NPC is one of the most aggressive ones, having one of the highest metastatic rates not only in the head and neck region, but also in the distant locations. Cutaneous metastases, although rare, can be found locally or regionally, including at the thorax level, making it necessary to differentiate from other tumors $(18,19)$. This type of carcinoma is not only capable of cell migration, invasion and metastasis, but its specific micro-environment may also determine various changes in other tissues, increasing the chance of a new cancer-type development $(12,20)$.

In the medical practice, the development of two different cancer types that are not linked in any way, has almost never been documented, although each time this occurs, the possible link needs to be investigated in depth.

NPC may influence the development of colorectal cancer (or vice-versa), especially if the host is predisposed to gene instability. These two types of malignant tumors have a series of common genes that can influence their progression.

Serine protease inhibitor kazal-type 6 (SPINK-6) is the main gene involved in the proliferation and metastasis development of NPC. SPINK-6 protein is part of the serine protease inhibitor kazal-type family, inhibiting the activity of the kallikrein (KLK) protease; it is a serine protease inhibitor selective for KLK. In metastatic cells, overexpression of SPINK-6, correlated with KLK protease inhibition was identified by Zheng et al (20). On the other hand, it has been proven that SPINK proteins are involved in the upregulation of colorectal 
cancer, promoting its growth, angiogenesis, migration and invasion $(21,22)$.

Bcl-2 mutation is associated both with colorectal cancer, and NPC $(23,24)$. The mutant $\mathrm{Bcl}-2$ gene is responsible for defective apoptosis, leading to an increased aggressive behavior of the tumor. Ismail et al (25) estimated that 30-94\% of colorectal cancer cases present abnormal Bcl-2 expression, inhibiting cell apoptosis and promoting tumor cell development and progression. On the other hand, the same mutation has been found in $90-100 \%$ of NPC cases. Bcl-2 overexpression in this type of cancer is directly correlated to its aggressive behavior (26).

It is less likely that concomitant Bcl-2 mutation development in the two different types of cells (colorectal and nasopharyngeal ones) are independent of each other. The Bcl-2 mutation in the colorectal tissue determines changes in the microenvironment, which in turn can determine Bcl-2 mutation in the nasopharyngeal tissue (or vice versa), especially in a patient whose genes are unstable and predisposed to mutations.

This case's particularity stems from the development of a metachronous tumor, i.e., a rectal adenocarcinoma and NPC, two malignant tumors that have different prognosis and progression. A noteworthy fact concerns paraneoplastic syndromes that may take form in head and neck carcinomas, as in the case of squamous cell carcinoma. The syndromes may be identified as a preceding, concurrent or subsequent manifestation of malignancy, as a result of tumor-producing protein hormones, enzymes or fetal proteins, tumor-induced antibody production or cytokine production.

The paraneoplastic syndromes, which can be found in squamous cell carcinoma of the head and neck region, can be classified as follows: i) endocrine manifestations, ectopic antidiuretic hormone secretion [leading to the syndrome of inappropriate antidiuretic hormone secretion (SIADH)], humoral hypercalcemia of malignancy (HHM) with hypercalcemia complications, ectopic adrenocorticotropic hormone (ACTH), ginecomastia; ii) neurological and muscular manifestations in the form of subacute cerebellar degeneration developing as tumor-producing antineural antibodies that determine neuronal death, Eaton-Lambart myasthenic syndrome, paraneoplastic encephalomyelitis with anti-Hu antibodies, and limbic encephalitis; iii) ophthalmic manifestations, i.e., photophobia due to auto-antibodies against retinal tissue with apoptosis; iv) rheumatologic manifestations including polyarthritis, polymyalgia rheumatica, and hypertrophic osteoarthropathy; v) dermatological manifestations such as itching, alopecia, herpes zoster, acanthosis nigricans, acrokeratosis paraneoplastica (also known as Bazex syndrome), Sweet's syndrome (also known as, acute febrile neutrophillic dermatosis), digital necrosis, paraneoplastic pemphigus, and vitiligo (as part of the Cowden syndrome, but mainly develops in regards to thyroid carcinoma, in which case the patient needs to be further investigated for other autoimmune-type diseases); vi) hematological manifestations including non-bacterial thrombotic endocarditis with venous thrombosis and arterial emboli, paraneoplastic polyvasculitis, hypereosinophilia and itching accompanying thrombocytosis, anemia, erythrocytosis, disseminated intravascular coagulation (DIC), and leukemoid reactions (immature white blood cells in the bloodstream) (27-31).
It is presumed that cavum tumor development is linked to the vicious handling of the hearing aid, lack of local hygiene or hearing aid hygiene, and to aggressive endauricular maneuvers. Another hypothesis is based on the existence of multiple, chronic exudative, relapsing otitis media that were therapeutically neglected. The risk factors that are also present in this patient are: Age, sex, decreased immunity, other comorbidities and a highly probable positive EBV status. A possibly important risk factor was antihypertensive drug use; including hydrochlorothiazide, statins are incriminated in non-melanoma skin cancer development (such as basal and squamous cell carcinoma, 2 types of cancer in the development of which another drug class is used, e.g., tetracyclines) (32-34).

Thus, as stated previously, it is highly probable that the two malignant tumors (developed in the same patient, at different time intervals and with such different locations and morphology) may have a linking genetic component. This atypical and highly interesting case presented clinical and imaging characteristics which uphold the idea that this very rare type of tumor is difficult to diagnose in its early stages, a reason for applying treatment frequently as palliative care. In such cases, psychological support based on physician trust and open communication is one of the ways of tilting the scale towards recovery and long-term survival. In Romania, individual and (support) group psychotherapy is still not given the proper place it needs in patient management (35).

In summary, research is still open in what concerns the role played by Bcl-2 and SPINK6 in tumor promotion, the therapeutic management options and the ways in which they can be identified as early stage, taking into consideration that most patients suffering from this malignancy are mainly from the rural environment, with little medical education, a high number of comorbidities, and who frequently ignore the signs and symptoms and their eventual treatment, steps which could be of vital importance to their survival.

\section{Acknowledgements}

Not applicable.

\section{Funding}

The present study was supported by 'Dunarea de Jos' University of Galati (internal grant no. RF3668/01.10.2021).

\section{Availability of data and materials}

The information generated and analyzed during the current study is available from the corresponding author on reasonable request.

\section{Authors' contributions}

DJ(S), ALT, ML, EN, CO, LR, AME, CB and LA have contributed to the acquisition, analysis and interpretation of data and they have supervised and substantially revised this work; they have substantial contributions to the conception and design of the work. All authors had equal participation, equal contributions and the same rights to this article. All authors have read and agreed to the published version of the 
manuscript. DJS and EN confirm the authenticity of the raw data.

\section{Ethics approval and consent to participate}

Ethics approval and consent to participate were granted by the 'Dunarea de Jos' University's Ethics Committee with the decision number 331 from 15.04.2021.

\section{Patient consent for publication}

Patient consent was granted and is part of the patient's personal chart.

\section{Competing interests}

The authors declare to not have competing interests.

\section{References}

1. Wu L, Li C and Pan L: Nasopharyngeal carcinoma: A review of current updates. Exp Ther Med 15: 3687-3692, 2018.

2. Spano JP, Busson P, Atlan D, Bourhis J, Pignon JP, Esteban C and Armand JP: Nasopharyngeal carcinomas: An update. Eur J Cancer 39: 2121-2135, 2003.

3. Thompson LD: Update on nasopharyngeal carcinoma. Head Neck Pathol 1: 81-86, 2007.

4. Tatu A, Radaschin D, Constantin V, Stana P and Ardeleanu V: Laser therapy in superficial morphea lesions-indications, limitations and therapeutic alternatives. J Mind Med Sci 7: 46-51, 2020

5. Draganescu M, Baroiu L, Iancu A, Dumitru C, Radaschin D, Polea ED, Bobeica C, Tatu AL, Niculet E and Fekete GL: Perspectives on skin disorder diagnosis among people living with HIV in southeastern Romania. Exp Ther Med 21: 97, 2021.

6. Rebegea LF, Firescu D, Dumitru M and Patrascu A: Skin spiradenocarcinoma-case presentation. Rom J Morphol Embryol 57: 327-330, 2016

7. Rebegea LF, Dumitru M, Serban C, Firescu D, Ivan I, Craescu M and Romila A: Survival and toxicity after treatment with sorafenib in unresectable hepatocellular carcinoma. Acta Medica Mediterranea 4: 2113-2118, 2019.

8. Craescu M, Rebegea L, Ivan I, Dumitru M, Serban C and Firescu D: Therapeutic challenges in a case of trachea neuroendocrine tumor. Acta Medica Mediterranea, 3: 1493, 2019.

9. Tatu AL,Elisei AM, Chioncel V, Miulescu M and Nwabudike LC: Immunologic adverse reactions of $\beta$-blockers and the skin (Review). Exp Ther Med 18: 955-959, 2019.

10. EI-Naggar AK, Chan JKC, Grandis JR, Takata $T$ and Slootweg PJ (eds): World Health Organization Classification of Head and Neck Tumours. IARC Press, Lyon, France, pp.65-70, 2017.

11. Weiland LH: The histopathological spectrum of nasopharyngeal carcinoma. IARC Sci Publ: 41-50, 1978.

12. Nwabudike LC and Tatu AL: Reply to Gambichler T, et al: Altered epigenetic pathways and cell cycle dysregulation in healthy appearing skin of patients with koebnerized squamous cell carcinomas following skin surgery. J Eur Acad Dermatol Venereol 33: e3-e4, 2019.

13. Nwabudike LC and Tatu AL: Reply to Happle R, et al: Koebner's sheep in Wolf's clothing: Does the isotopic response exist as a distinct phenomenon? J Eur Acad Dermatol Venereol 32: e336-e337, 2018.

14. Ardeleanu V, Georgescu C, Frîncu LD, Frîncu LL and Vesa D: Angiogenesis as prospective molecular biology technique for cancer study. Rom Biotechnol Lett 19: 9637-9648, 2014.

15. Niculet E, Chioncel V, Elisei AM, Miulescu M, Buzia OD, Nwabudike LC, Craescu M, Draganescu M, Bujoreanu F, Marinescu E, et al: Multifactorial expression of IL-6 with update on COVID-19 and the therapeutic strategies of its blockade (Review). Exp Ther Med 21: 263, 2021.
16. Muzaffar R, Vacca F, Guo H, Mhapsekar R and Osman M: Pediatric nasopharyngeal carcinoma as seen on $18 \mathrm{~F}-\mathrm{FDG}$ PET/CT. Front Oncol 9: 110, 2019.

17. Looi L: Tumor-associated tissue eosinophilia in nasopharyngeal carcinoma. A pathologic study of 422 primary and 138 metastatic tumors. Cancer 59: 466-470, 1987.

18. Tatu AL: Umbilicated blue black lesion on the lateral thorax. J Cutan Med Surg 21: 252, 2017.

19. Tatu AL: Black nodule on the forearm. J Cutan Med Surg 21: $157,2017$.

20. Zheng LS, Yang JP, Cao Y, Peng LX, Sun R, Xie P, Wang MY, Meng DF, Luo DH, Zou X, et al: SPINK6 promotes metastasis of nasopharyngeal carcinoma via binding and activation of epithelial growth factor receptor. Cancer Res 77: 579-589, 2016.

21. Gouyer V, Fontaine D, Dumont P, de Wever O, Fontayne-Devaud H Leteurtre E, Truant S, Delacour D, Drobecq H, Kerckaert JP, et al: Autocrine induction of invasion and metastasis by tumor-associated trypsin inhibitor in human colon cancer cells. Oncogene 27: 4024-4033, 2008.

22. Lu X, Lamontagne J, Lu F and Block TM: Tumor-associated protein SPIK/TATI suppresses serine protease dependent cell apoptosis. Apoptosis 13: 483-494, 2008.

23. Testa U, Pelosi E and Castelli G: Colorectal Cancer: Genetic abnormalities, tumor progression, tumor heterogeneity, clonal evolution and tumor-initiating cells. Med Sci (Basel) 6: 31, 2018.

24. Dong SS, Wang N, Yang CP, Zhang GC, Liang WH, Zhao J and Qi Y: Giant cell-rich solitary fibrous tumor in the nasopharynx: Case report and literature review. Onco Targets Ther 13: 6819-6826, 2020.

25. Ismail NI, Othman I, Abas F, Lajis NH and Naidu R: Mechanism of apoptosis induced by curcumin in colorectal cancer. Int J Mol Sci 20: 2454, 2019

26. Geramizadeh B, Marzban M and Churg A: Role of immunohistochemistry in the diagnosis of solitary fibrous tumor, a review. Iran J Pathol 11: 195-203, 2016.

27. Mathew DG, Rooban T, Janani V, Joshua E, Rao U and Ranganathan K: Review of paraneoplastic syndromes associated with oropharyngeal squamous cell carcinoma. J Oral Maxillofac Pathol 14: 41-47, 2010.

28. Tatu AL and Nwabudike LC: The treatment options of male genital lichen sclerosus et atrophicus short title for a running head: Treatments of genital lichen sclerosus. In: Proceedings of the 14th National Congress of Urogynecology and the National Conference of the Romanian Association for the Study of Pain, pp262-264, 2017.

29. Tatu AL and Ionescu MA: Multiple autoimmune syndrome type III-thyroiditis, vitiligo and alopecia areata. Acta Endocrinol (Buchar) 13: 124-125, 2017.

30. Mihăilă B, Dinică RM, Tatu AL and Buzia OD: New insights in vitiligo treatments using bioactive compounds from Piper nigrum. Exp Ther Med 17: 1039-1044, 2019.

31. Alter M, Mengoni M and Gaffal E: Cutaneous manifestations of internal malignancy. J Dtsch Dermatol Ges 18: 456-469, 2020.

32. Tatu AL, Ciobotaru OR, Miulescu M, Buzia OD, Elisei AM, Mardare N, Diaconu C, Robu S and Nwabudike LC: Hydrochlorothiazide: Chemical structure, therapeutic, phototoxic and carcinogenetic effects in dermatology. Rev Chim 69: 2110-2124, 2018

33. Nwabudike LC, Elisei A, Buzia OD, Miulescu M and Tatu A: Statins. A review on structural perspectives, adverse reactions and relations with non-melanoma skin cancer. Rev Chim 69: 2557,2018

34. Nwabudike LC and Tatu AL: Response to-Chronic exposure to tetracyclines and subsequent diagnosis for non-melanoma skin cancer in a large Mid-Western US population. J Eur Acad Dermatol Venereol 32: e159, 2018.

35. Rebegea L, Firescu D, Baciu G and Ciubara A: Psycho-oncology support. Brain 10: 77-88, 2019.

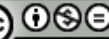

This work is licensed under a Creative Commons Attribution-NonCommercial-NoDerivatives 4.0 International (CC BY-NC-ND 4.0) License. 